\title{
Long term treatment with betaine in methylenetetrahydrofolate reductase deficiency
}

\author{
Eric Ronge, Bengt Kjellman
}

Department of

Paediatrics, Central

Hospital Skövde,

Sweden

E Ronge

B Kjellman

Correspondence to: Dr Eric Ronge, Department of Paediatrics, Central

Hospital, S-541 85 Skövde, Sweden.

Accepted 5 October 1995

\begin{abstract}
A girl aged 7.5 years with deficiency of 5,10-methylenetetrahydrofolate reductase was treated from early infancy with betaine, 3-6 g daily. She has slight microcephaly, moderate developmental delay, and impaired vision but there have been no obvious signs of folate deficiency. From 4 years of age, she developed an unexplained extreme increase in appetite and weight. Recent magnetic resonance imaging of her brain was normal. The plasma methionine levels have been normal but in the lower range, and the total plasma homocysteine concentrations have been moderately increased (54 to 85 $\mu \mathrm{mol} /$ ) without obvious correlation with the different betaine doses given. Folic acid has sometimes been added.

(Arch Dis Child 1996; 74: 239-241)
\end{abstract}

Keywords: 5,10-methylenetetrahydrofolate reductase deficiency, long term treatment, betaine, 5-methyltetrahydrofolate.

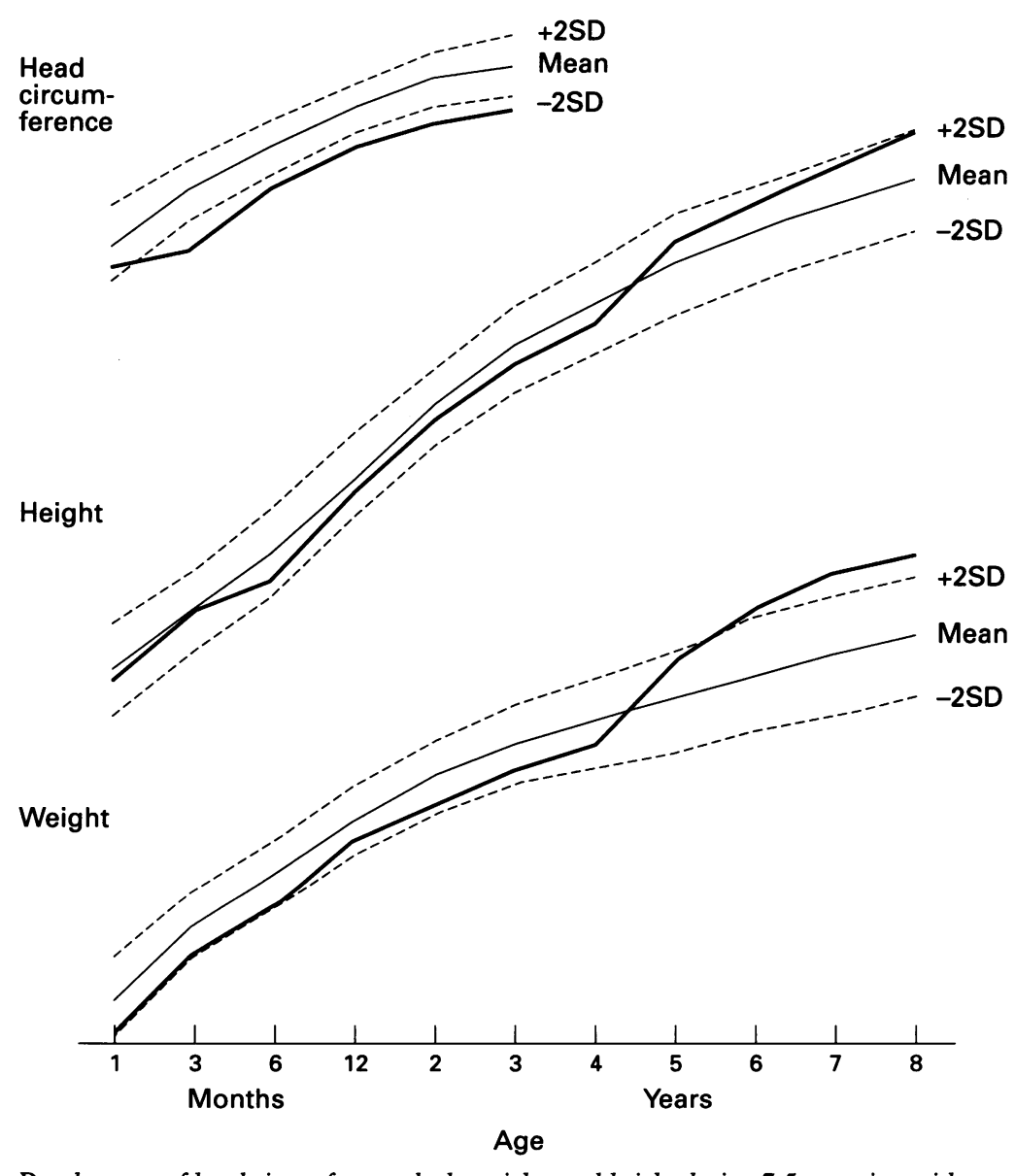

Development of head circumference, body weight, and height during 7.5 years in a girl with severe deficiency of MTHFR and treated with betaine in doses of 3-6 $\mathrm{g}$ daily.
One of the causes of homocystinuria is deficient activity of 5,10-methylenetetrahydrofolate reductase (MTHFR). This inborn error of folate metabolism sometimes presents in early infancy with progressive severe neurological symptoms from demyelination caused by hypomethioninaemia. ${ }^{1-3}$ The enzymatic block can be bypassed by a secondary pathway using betaine (trimethylglycine) as a substrate. ${ }^{2-5} \mathrm{We}$ have previously reported on a girl with severe MTHFR deficiency who, during the first month of life, developed life threatening symptoms but recovered on a regimen of methionine for one week and betaine for continuous use. ${ }^{4}$ We report here on her clinical development, treatment with betaine, and biochemical responses up to the age of $7 \cdot 5$ years.

\section{Case report}

Her growth pattern is shown in the figure. At the age of 4 years the girl's previously poor appetite increased. Growth accelerated and her present weight now corresponds to the mean +3 SD for healthy children. Head circumference was normal at birth, but there was no increase during the first two months of life and no catch up phase after treatment.

She sat without support at 10 months and started to walk at 17 months. She spoke two word sentences at 3 years. She still has slight muscle hypotonia, but no atrophy, spasticity, or obvious ataxia. She is a little clumsy but her fine motor skills are adequate. Her hearing is normal. Her visual acuity is decreased to $6 / 18$ bilaterally using glasses. She has a bilateral convergent squint and nystagmus, but normal pupils, media, retinas, and optic discs. Psychological assessments according to Griffith at $4 \cdot 5,5 \cdot 5$, and $7 \cdot 5$ years of age show results corresponding to 2 years, 3 years, and 5 years of age respectively, with an even profile.

Her general health has been unremarkable, but during the first three years of life she was lethargic the day before respiratory infections; biochemical analyses produced no explanation for this. Computed tomography of the brain was normal in the acute phase of the disease. At $7 \cdot 5$ years magnetic resonance imaging (MRI) of the brain showed no abnormality. A recent electroencephalogram was normal.

During the acute stage, there was a high urine homocystine concentration (230 $\mathrm{mmol} / \mathrm{mol}$ creatinine) and plasma methionine was below detectable limits. The values returned to normal after treatment. The total plasma homocysteine concentration - that is, the combined free and protein bound fractions - estimated at 9 months was $78 \mu \mathrm{mol} / 1$ (reference approximately $4-18 \mu \mathrm{mol} / \mathrm{l}$ ) during 
Doses of betaine, total plasma homocysteine, and plasma methionine during the seven years of follow up. At 3.5 years of age, a lower dosage and a higher dose of betaine were tested

\begin{tabular}{lcll}
\hline Age & $\begin{array}{l}\text { Dose of } \\
\text { betaine }(g)\end{array}$ & $\begin{array}{l}\text { Total p-homocysteine } \\
(\mu \text { molh })\end{array}$ & $\begin{array}{l}p \text {-Methionine } \\
(\mu \text { molh })\end{array}$ \\
\hline 9 months & 3 & 78 & 21 \\
12 months & 6 & 70 & 24 \\
13 months & 6 & 70 & 45 \\
14 months & 6 & 62 & 24 \\
2 years & 6 & 73 & 19 \\
3.5 years & 6 & 77 & 17 \\
3.5 years & 6 & 84 & 13 \\
3.5 years & 3 & 83 & 14 \\
3.5 years & 10 & 67 & 21 \\
5 years & 6 & 85 & 29 \\
7.5 years & 6 & 54 & 19 \\
\hline
\end{tabular}

treatment with $3 \mathrm{~g}$ betaine daily, and decreased to only $70 \mu \mathrm{mol} / 1$ on $6 \mathrm{~g}$ betaine daily. The table shows the results of the analyses of total homocysteine and methionine in plasma in relation to the daily doses of betaine during follow up. At 3.5 years of age a reduced daily dose of betaine of $3 \mathrm{~g}$ and an increased dose of $10 \mathrm{~g}$ were tested, without any significant changes in biochemical values. She was also given $15 \mathrm{mg}$ folic acid daily up to the age of 2 years and 5 mg daily from the age of 5 years because of the risk of folate deficiency. Analyses of the bone marrow and red cell folate have been quite normal and we have detected no signs of neuropathy.

\section{Discussion}

As far as we know, this is the first report of a case with severe deficiency of MTHFR ( $2 \%$ residual enzyme activity) associated with serious progressive symptoms during the first month of life, successfully treated with betaine and methionine initially and with betaine continuously for several years. In a previous review, two older infants are mentioned who died after four days and three weeks of treatment with betaine. ${ }^{2}$ Brandt et al reported two infants with MTHFR deficiency treated with betaine (one before the appearance of symptoms) who had normal development. ${ }^{5}$ In children with long lasting neurological symptoms before treatment, the biochemical response to betaine is good, but clinical improvement is limited. ${ }^{3}$ We think that the non-progressive mild mental and motor retardation in our patient is due to irreversible brain damage caused by demyelination during the time she was severely methionine deficient, but we cannot rule out hypoxia. A recent MRI scan of the brain showed normal myelination while on treatment, which has been seen in similar cases. ${ }^{6}$ Impaired vision is thought to be due to damage to the visual cortex, but no corresponding lesion was found on MRI. Nor did MRI explain the large increase in appetite, weight, and height appearing at age 4 and still present. This suggests diencephalic damage analogous to that caused by lesions in the hypothalamic area or in some metabolic diseases. ${ }^{7} 8$

In MTHFR deficiency with onset in early infancy, immediate restoration of plasma methionine is the primary goal. This can be done quickly by giving pure methionine by mouth, but treatment with betaine should also start at once in order to obtain a more stable and continuous normal methionine level and suppress homocysteinaemia. The optimum dose of betaine is difficult to determine because of limited experience and the heterogeneity of the disease. By monitoring plasma methionine and homocysteine concentrations, we found that 3-6 $\mathrm{g}$ of betaine daily $(2 \times 3 \mathrm{~g})$ might be enough; $10 \mathrm{~g}$ betaine was tested but there was no obvious clinical or biochemical improvement and it was very difficult to administer because of the unpleasant taste and gastric irritation. A doseresponse relation between betaine and plasma methionine has been shown by Wendel et al. ${ }^{3}$ Complete suppression of raised plasma homocysteine levels is not usually achieved by betaine treatment, which implies a risk of thrombovascular or neurotoxic effects and other homocysteine related diseases. ${ }^{9}$ We intend to increase the betaine dose gradually to $12 \mathrm{~g}$, although this is unlikely to eliminate future morbidity. We are also considering methionine supplementation as the plasma concentrations of methionine are sometimes low.

Serum and red cell folate levels are usually low in severe MTHFR deficiency because of decreased formation of 5-methyltetrahydrofolate (5-methyl-THF), which represents the active and predominant form of folate in blood and brain. Successful treatment with high doses of folic acid has been reported in some cases, but it probably will not help our patient, who has very low MTHFR activity, producing only small amounts of 5-methyl-THF. ${ }^{10}$ Furthermore, a folic acid load may be harmful because of accumulation of pre-block metabolites with inhibitory effects on other folate pathways. ${ }^{2} 5$-Methyl-THF is the only folate metabolite that crosses the blood-brain barrier, which explains the common finding of low levels of 5-methyl-THF in the cerebrospinal fluid in MTHFR-deficient patients. In the brain, 5-methyl-THF is used as a methyl group donor to maintain the remethylation of homocysteine to methionine, crucial for the myelination and synthesis of neurotransmitters. Methyl groups can also be delivered by betaine, thus compensating for the lack of 5methyl-THF and allowing recycling of methionine and normal myelination. The synthesis of serotonin and perhaps dopamine may depend on the presence of both methionine and 5methyl-THF. ${ }^{11}$ This may limit the therapeutic use of betaine and may explain the abnormal appetite and growth in our patient. We are now considering starting supplementation with 5methyl-THF despite suggestions that the source has to be endogenous. ${ }^{2}$

The parents of our patient have decided not to have any more children. In the event of pregnancy in a family with a previously affected child, a plan must be established for prenatal or immediate postnatal diagnosis and immediate treatment with methionine and betaine.

We thank Drs M Azam Mansoor (the University of Bergen, Norway), B Hultgren (University of Lund, Sweden), and E Holme (University of Gothenburg, Sweden) for the biochemical analyses. We also thank Dr Fleming Skovby of the 
Rigshospital in Copenhagen, Denmark, and Dr L Brattström of the Central Hospital in Kalmur, Sweden, for valuable discussion and correspondence.

1 Mudd SH, Uhlendorf BW, Freeman JM, Finkelstein JD, Shih VE. Homocystinuria associated with decreased Biophys Res Commun 1972; 46: 905-12.

2 Erbe RW. Folates and pterins: nutritional, pharmacological and physiological aspects. In: Blakley RL, Whitehead and physiological aspects. In: Blakley RL, Whitehead VM, eds. Inborn errors of folate

3 Wendel U, Bremer HJ. Betaine in the treatment of homocystinuria due to 5,10-methylenetetrahydrofolate reductase deficiency. Eur $\mathcal{F}$ Pediatr 1984; 142: 147-50.

4 Holme E, Kjellman B, Ronge E. Betaine for treatment of homocystinuria caused by methylenetetrahydrofolate reductase deficiency. Arch Dis Child 1989; 64: 1061-4.

5 Brandt NI, Christensen E, Skovby F, Diernes B. Treatment of methylene-tetrahydrofolate reductase from the neonatal period. Amersfoort, The Netherlands: The Society for Study of Inborn Errors of Metabolism 1986: 23.

6 Surtees R, Leonard J, Austin S. Association of demyelination with deficiency of cerebrospinal fluid S-adenosylmethionine in inborn errors of methyl-transfer pathway. Lancet 1991; ii: $1550-4$.

7 Gamstorp I, Kjellman B, Palmgren B. Diencephalic syndromes of infancy. F Pediatr 1967; 70: 383-90.

8 Kjellman B, Gamstorp I, Brun A, Öckerman P-A, Palmgren B. Mannidosis: a clinical and histopathologic study. $\mathfrak{f}$ Pediatr 1969; 75: 366-73.

9 Brattström L, Hardebo J-E, Hultberg B. Moderate homocysteinemia - a possible risk factor for arteriosclerotic cystebrovascular disease. Stroke 1984; 15: 1012-6.

10 Takenaka T. Effect of folic acid for treatment of homocystinuria due to 5,10-methylenetetrahydrofolate reductase deficiency. Rinsho Shinkeigaku 1993; 33: 140-5.

11 Surtees R, Heales S, Bowron A. Association of cerebrospinal fluid deficiency of 5-methyltetrahydrofolate, but not $\mathrm{S}$-adenosyl methionine, with reduced concentrations of the acid metabolites of 5-hydroxytryptamine and dopamine. Clin Sci 1994; 86: 697-702.

\section{Vitamin $A$ and immunisation}

The saga of vitamin supplementation for children in developing countries (see Archivist 1991, page 139 and 1992, pages 288 and 1441) continues to develop. A study carried out in Indonesia (Lancet 1995; 345: 1330-2) has shown that vitamin A given at the same time as measles vaccine may blunt the response to the vaccine.

In the Bogor district of West Java there is thought to be a high incidence (more than $50 \%$ of infants) of subclinical vitamin A deficiency. Three hundred and thirty six 6 month old infants were immunised with Schwarz measles vaccine and at the same time received randomly either 100000 IU of vitamin A or placebo capsules. A vaccine induced measles rash was less common after vitamin A (9\%) than after placebo (16\%) suggesting that the vitamin might restrict the proliferation of the vaccine virus. Two thirds of all babies were found to have maternally derived measles antibody (titre of more than 1 in 8) at the time of immunisation. In these babies the seroconversion rate was $66 \%$ in the vitamin A group and $79 \%$ in the placebo group $(p<0.04)$ whereas it was $100 \%$ in babies without maternal antibody irrespective of whether they were given the vitamin or not. Among infants with significant maternal antibody those given vitamin A had a risk ratio of 1.62 for not seroconverting and of 1.72 for not attaining a protective titre (greater than 1 in 120) compared with those given placebo. Mean titres of measles antibody were lower at one and six months after immunisation in the vitamin A group.

Vitamin $A$ is known to enhance immune responses and it is suggested that this effect, together with pre-existing antibody, inhibits the replication of live vaccine virus and therefore results in a diminished vaccine response. Giving vitamin A along with immunisation obviously has considerable logistic advantage. Delaying measles immunisation to 9 months when fewer children will still have maternal antibody might lead to better vaccine responses but there are no data to confirm this, neither is it known whether the reduced antibody response at 6 months of age in those given simultaneous vitamin $\mathrm{A}$ is offset by the beneficial effect of the vitamin on morbidity and mortality in general. It has been suggested that vitamin supplementation might be given with the earlier diphtheria, pertussis, tetanus and oral polio immunisations, but the work in Indonesia raises doubt about such a policy especially as regards the possible effect on live oral polio vaccination (see commentary by Ross, Lancet 1995; 345: 1317-8). Both vitamin A supplementation and immunisation are clearly extremely important. Much more work needs to be done to clarify their interaction and the best ways of delivering both to children at risk. 